\title{
Prospect of 3D Warp Knitted Spacer Fabric and its Effect on Pressure Relieve for Reducing the Prevalence of Pressure Ulcers for Immobile Patients
}

Shuvo II*, Chakma K and Toutant D

Department of Biosystems Engineering, University of Manitoba, Canada

\begin{abstract}
Many hospitals use paper thin bed sheets with high friction coefficients which are not ideal for patients with pressure ulcers and who are at risk of developing. These patients suffer a great deal of pain, which could have been prevented. Lying on a weak bed sheet with no regards to regulating micro-climate is a clear promoter of pressure ulcers. Another key factor of a hospital bed sheet is they are to be easily washed or disposed of because of all the unknown fluids that could seep onto the sheet. Therefore, the sheet must not only be to comfort those with pressure ulcers but to be easily washable and reusable. Again, in a hospital setting being able to easily wash the sheet and for it to hold its form is significant for reducing the cost of throwing away sheets less often.

Therefore a theory has been proposed to design a 3D knit spacer bed sheet that will allow patients with pressure ulcers to be comfortable by ensuring a low friction coefficient between their skin and the material. The friction coefficient will be reduced by not only the structure but by the 70 percent polyester, 22 percent polypropylene and eight percent spandex blend. The friction coefficient will stay low due to a high wicking and evaporation capability to ensure the skin stays dry as well as the material. The 3D knit spacer bed sheet also has a higher compressibility which distributes pressure more evenly as well as enabling a care giver to easily rotate an immobile person into a new position. The proposed bed sheet will be easily washable to ensure all bodily fluids such as vomit, blood, and others have been removed. This blanket will be slightly more expensive but is expected to last longer than a typical hospital bed sheet.
\end{abstract}

Keywords: Pressure ulcer; Immobility; Spacer fabric; 3D warp knit

\section{Introduction}

\section{Purpose}

The purpose is to create a bed sheet which reduces the discomfort to those who have pressure ulcers as well as significantly reduce the risk of developing a pressure ulcer. Many hospitals use paper thin bed sheets with high friction coefficients which are not ideal for patients with pressure ulcers and who are at risk of developing. These patients suffer a great deal of pain, which could have been prevented. Lying on a weak bed sheet with no regards to regulating micro-climate is a clear promoter of pressure ulcers. Another key factor of a hospital bed sheet is they are to be easily washed or disposed of because of all the unknown fluids that could seep onto the sheet. Therefore, the sheet must not only be to comfort those with pressure ulcers but to be easily washable and reusable. Again, in a hospital setting being able to easily wash the sheet and for it to hold its form is significant for reducing the cost of throwing away sheets less often.

\section{Problem}

Pressure ulcers are a preventable but growing problem. Every year roughly 60,000 people die due to a direct result of pressure ulcers and are only growing [1]. There is many factors which pertain to the development of pressure ulcers from a bed sheet. These include and are not limited by having a high friction coefficient from not only the material but by the wrinkles made from tossing and turning. These wrinkles due to additional friction to the area exposed to the wrinkled area and are generally located next to a bony part of the body where pressure ulcers are commonly formed [2]. Other problems of typical hospital bed sheets consist of regulating the micro-climate and ensuring regulated moisture content. The micro-climate ensures the sheet has sufficient air permeability to help in regulating temperature which in turn reduces perspiration and regulating the moisture moves through the fabric is extremely important because if the fabric is wet the frictional coefficient value doubles [3]. Therefore, the bed sheet must have a high wicking value but a reasonably high evaporation rate to disperse of the moisture. All factors is considered, the bed sheet will greatly aid against the protection of developing and existing pressure ulcers.

\section{Scope}

The 3D knit spacer bed sheet will allow patients with pressure ulcers to be comfortable by ensuring a low friction coefficient between their skin and the material. The friction coefficient will be reduced by not only the structure but by the 70 percent polyester, 22 percent polypropylene and eight percent spandex blend. The friction coefficient will stay low due to a high wicking and evaporation capability to ensure the skin stays dry as well as the material. The 3D knit spacer bed sheet also has a higher compressibility which distributes pressure more evenly as well as enabling a care giver to easily rotate an immobile person into a new position. The proposed bed sheet will be easily washable to ensure all

*Corresponding author: Ikra Iftekhar Shuvo, Graduate Research Assistant Department of Biosystems Engineering, University of Manitoba, Canada, Tel: 2044047633; E-mail: iishuvo123@gmail.com

Received January 16, 2018; Accepted January 18, 2018; Published January 30, 2018

Citation: Shuvo II, Chakma K, Toutant D (2018) Prospect of 3D Warp Knitted Spacer Fabric and its Effect on Pressure Relieve for Reducing the Prevalence of Pressure Ulcers for Immobile Patients. J Textile Sci Eng 8: 335. doi: 10.4172/21658064.1000335

Copyright: (c) 2018 Shuvo II, et al. This is an open-access article distributed under the terms of the Creative Commons Attribution License, which permits unrestricted use, distribution, and reproduction in any medium, provided the original author and source are credited. 
bodily fluids such as vomit, blood, and others have been removed. This blanket will be slightly more expensive but is expected to last longer than a typical hospital bed sheet.

\section{Proposal}

\section{Need for the research and development activity}

A pressure ulcer is a type of chronic wound that is also known as bedsores, pressure sore, or decubitus ulcer and can be defined as "a wound that develops as a result of pressure on a bony prominence that leads to soft tissue damage" [4]. Basal and Ilgaz also stated: "pressure ulcers are localized areas of tissue damage that develop due to pressure usually over a bony prominence". Zhong et al. conducted a pilot research and derived an experimental result with the immobility of patient's being the major factor which facilitates the formation of pressure ulcers along with skin disorder; and this immobility would engender the long-term friction, shear, and pressure, of human skin toward bed sheet fabric that could ultimately be responsible for the pressure ulcer and skin problems [2]. When immobile patients are in bed, their skin experiences long hours under pressure and friction engendered by textile bed linens; which may give rise to pressure ulcers. Due to long-term pressure imposed on the skin and tissues beneath it, proper circulation of oxygen and nutrient supply is obstructed that propagates deep ulcers; whereas shearing and friction engender the stretching of human skin along with twisting of blood vessels, which are also the prime reasons for pressure ulcers [5]. Lack of proper moisture and air permeability management system of the microclimate surrounding the human skin is also a major factor as wetness makes human skin soft and reduces the tolerance level of tissues to shear and friction making it vulnerable to ulcer $[2,5]$.

In current medical textiles market, single or two-ply bed sheets composed of $50 \%$ cotton and $50 \%$ polyester (PET or polyethylene terephthalate) are used. These bed sheets are in direct contact with the patient's skin which is prone to be contaminated by pressure ulcer. However, there are various limitations of this one to two-ply 50 percent cotton and 50 percent polyester bed sheets aside from the comfort which cannot reduce the prevalence of pressure ulcer. These bed sheets must not only provide relief but prevent from forming pressure ulcers too. This entitles a bed sheet with a lower friction coefficient as well as a higher strength and or elasticity to protect against wrinkling. Wrinkles in a bed sheet are not ideal for pressure ulcers because of the extra friction created by the creasing of the fabric. In addition, it is difficult to move and rotate without creating creases due to the low compressibility of the one to two-ply fabrics. Having some compressibility in a bed sheet would also greatly increase the pressure distribution evenly across a patient's body rather than be amplified by whichever part is putting the most force on a certain location of the sheet. Another key limitation of the current bed sheet is the micro-climate between the sheet and the skin must be at a proper equilibrium to ensure the skin is not moist but not excessively dry. The current sheet can be overwhelmed by moisture at times and not able to evaporate it as fast as it is being absorbed which can lead to the moist skin which allows for an easier time to shear the skin as well as increases the risk of pressure ulcers. The air permeability can greatly reduce the amount of perspiration a patient is outputting due to the regulation of temperature not going to high; which is reduced in the traditional bed sheets due to a couple of reasons which include the tightly plain weave structure. However, such limitations can be easily solved by replacing it with the 3D warp knitted spacer fabric.

For the limitations discussed above with the existing products
( $50 \%$ cotton $+50 \%$ polyester bed sheets), the $3 \mathrm{D}$ warp knitted spacer fabric is chosen as it is a better choice in terms of pressure redistribution, reduced friction, and superior microclimate properties for its inherent enhanced comfort, moisture, and heat transport properties. The reason for using synthetic fibre (polyester, polypropylene, spandex) in the 3D spacer fabric is that synthetic fibres provide 50\%-60\% less coefficient of friction that traditional cotton bed sheets [6]. When the spacer fabric is compressed the spacer filament yarn are compressed. This eventually stretches fabric surface that leads to the compression of neighbouring filaments. Hence it spreads the force or load by the human body into a much larger area with a gradual transition to the neighbouring spacer filament yarns; which ultimately serve the purpose of pressure distribution or pressure relief [7]. On top of it, 3D spacer fabric is highly breathable, which in turn creates a moisture free environment for fast moisture transportation properties. This effect, in turn, reduces the chance of skin maceration [8]. Hence a higher or an increased level of comfort will be achieved by using $3 \mathrm{D}$ spacer fabric. Laundering of this 3D spacer will be quite easy as it consists of polyester which is wrinkle resistant and soil resistant up to a certain extent inherently. Presence of spandex and polypropylene will not cause any discrepancies for the ease of laundering. In addition to this, there will be less level of lint because cotton is not being used in this $3 \mathrm{D}$ spacer fabric.

\section{Research objectives}

- To produce 3D warp knitted spacer fabric to minimize friction/ shear and pressure on the skin.

- To find out the effect of 3D spacer fabric on pressure relieve and other advantages.

- To briefly discuss the advantages of using 3D warp knitted spacer fabric to be used in the hospital medical bed sheets instead of typical $50 \%$ cotton- $50 \%$ polyester fabric.

- To propose a new structural development that may optimize fabric performance.

- Testing of the 3D warp knitted to meet the standards and factors like pressure relief test, air permeability test, water vapour transmission, water absorption test, thermal conductivity test, bending \& compression test, stretch ability and recovery test, skin surface temperature change test, transepidermal water loss (TEWL) test, moisture management test, skin deformability tests, and coefficient of friction of human skin against sheet test.

\section{Methodology}

\section{Material}

3D spacer fabrics with the composition of the blend of polyester, polypropylene, and spandex, this production process has no adverse effect on the environment.

\section{Structures}

The top layer is made of $70 \%$ multifilament polyester (PET or polyethylene terephthalate) smooth thin yarn, $22 \%$ polypropylene yarn, and $8 \%$ spandex yarn; whereas bottom layer is made of $100 \%$ multifilament PET yarn. The composition of spacer yarn is $100 \%$ monofilament polyester yarn. The top and bottom layer will be joined together and kept apart by spacer yarn. Hence it is a sandwich textile structure with three layers. The knit structure for the top and the bottom layer is lock nit. For the top and bottom layers, there are 45 and 
Citation: Shuvo II, Chakma K, Toutant D (2018) Prospect of 3D Warp Knitted Spacer Fabric and its Effect on Pressure Relieve for Reducing the Prevalence of Pressure Ulcers for Immobile Patients. J Textile Sci Eng 8: 335. doi: 10.4172/2165-8064.1000335

26 wale $(5 \mathrm{~cm})$ and course $(5 \mathrm{~cm})$ or can be adjusted to convenience. The thickness of the $3 \mathrm{D}$ knitted fabric is $4 \mathrm{~mm}$.

The density of the spacer fabric is $205 \mathrm{~kg} / \mathrm{m}^{3}$ and number of spacer yarn is 180 spacers $/ \mathrm{cm}^{2}$. The yarn count for the top and the bottom layer is $32 \mathrm{Ne}$ and yarn count for monofilament is 50 dtex. Generally for spacer fabric thickness ranges from $1.5-60 \mathrm{~mm}$ and for sports $2.8-4$ $\mathrm{mm}[8]$.

\section{Method of production (Preparation)}

The fabric can be produced by using contemporary rib Rashcel warp knitting machine having two needle bars.

Method of characterization (Evaluation): The following evaluation tests will be conducted:

Pressure relief test: Any standard pressure pad consisting flexible film sensors can be used for instance Tekscan. Using the data from the pressure map pressure indices such as pressure peak (Pp), average pressure $(\mathrm{Pa})$ and contacting area $(\mathrm{Ac})$ can be calculated [7]. The lower value of $\mathrm{Pp}$ indicates the weakening of pressure on human skin by spacer fabric and vice versa. A higher value of Ac also indicates the weakening of pressure on human skin by spacer fabric and vice versa. Hence using the pressure peak $\mathrm{Pp}$, the pressure-relief property of spacer fabric will be evaluated.

Air permeability test: Air permeability of the fabric will be tested by KESF8-AP1 air permeability tester [8]. The ASTM D737-96 standard testing method needs to be followed.

Water vapour transmission through the material test: According to ASTM E96 standard method, this test will be conducted.

Moisture management test (MMT): AATCC-195 standard will be used by using the MMT tester as it is used to measure the liquid management properties of any technical fabric with great precision such us every parameter related the wicking behaviour instead of only vertical wicking test behaviour by traditional wicking test.

Water absorption test: We will do the water absorbency test according to ASTM D570.

Thermal conductivity test: The thermal conductivity will be tested by KES-F ThermoLabo II [8].

Bending and compression test: In order to measure the response to bending and compression properties of the sample fabric, the KES-F (Kawabata Evaluation System) will be used [8].

Stretchability and recovery test: The stretch and recovery properties of the spacer samples will be tested by INSTRON 4411 according to the British standard 4294.

Skin surface temperature change test: Skin surface temperature was measured in 0C (in degree Celsius) using a skin thermometer based on the infrared technique (Courage and Khazaka electronic $\mathrm{GmbH}$, Cologne, Germany) [6].

Transepidermal water loss (TEWL) test: TEWL will be measured using the Tewameter ${ }^{\circledast}$ TM 300, an open chamber device (Courage \& Khazaka electronic GmbH, Cologne, Germany) and expressed in g/ $\mathrm{m} 2 / \mathrm{h}$. Higher TEWL means higher the damage or impairment to the skin [6].

Skin deformability test: Using Cutometer ${ }^{\circledR}$ MPA 580 device, skin deformability will be measured with 'the corresponding software (Courage \& Khazaka Electronic GmbH, Cologne, Germany). Because it is an established method in skin research [6]. A pressure of $450 \mathrm{mBar}$ and a probe opening of $2 \mathrm{~mm}$ diameter will be used. Skin deformations will be measured by applying the negative pressure for $2 \mathrm{~s}$ and after releasing it for additional $2 \mathrm{~s}$ (accuracy: $\pm 3 \%$ ). The structural properties of skin will be expressed in terms of total extensibility (Uf, mm), and 'relative elastic recovery'(Ur/Uf) [6].

Co-efficient of frictions (COFs) of human skin against bed sheets evaluation: Co-efficient of frictions of human skin against bed sheet can be calculated using corneometry in combination with friction experiments on top of force plates [9].

\section{Research Challenge to be Addressed}

The human skin typically produces up to $1000 \mathrm{~cm}^{3} /$ hour perspiration to maintain a balanced body temperature of $33^{\circ} \mathrm{C}$ to $35^{\circ} \mathrm{C}$ [9]. This certain condition needs to be maintained while designing or manufacturing 3D spacer fabric to be used as medical bed sheets for the minimum level of comfort for the patients of pressure ulcer. And it is expected the porous structure of warp knit and 3D spacer structure will assist faster water vapour permeability; in order to reduce prevalence of ulcer by dissipate metabolic heat [5]. Even the coefficient of frictions (COFs) of human skin against medical bed sheets differs from the moisture condition of skin; such as COFs may increase from very dry to normally moist skin by 33\% [9]. Hence the 3D fabric should possess low coefficient of friction of fabric surface to facilitate the patients to change their body positions smoothly with less friction \& shear which can be achieved due to its superior porous knit structure, elastomeric spandex yarn and pressure redistribution mechanism [2,5]. Pressure between bony prominence of human skin and external surface such as, bed sheets shouldn't exceed $33 \mathrm{~mm} \mathrm{Hg}$ (mercury) pressure range in order prevent pressure ulcer; which is also a major challenge to be maintained by the 3D spacer fabric [10]. Application of innovative idea to lead the new knowledge and technology development:

1. The reason behind using a multifilament PET in the top and bottom surface instead of monofilament is that these multifilament yarns will enable easy gliding impart soft contact to human skin for improved comfort properties. In short, it will provide low friction or shear to human skin [11].

2. The current density of commercial 3D warp knit spacer fabric is approx. $55 \mathrm{~mm}$. However, in this project, the density is kept $205 \mathrm{~kg} /$ $\mathrm{m}^{3}$. Higher the density, higher the thermal conductivity and faster the heat transfer [8].

3. Polypropylene has been imparted for high wicking capacity to keep the human skin as dry as possible; which is rarely used in current commercial 3D spacer fabric.

4. Monofilament is used as spacer yarn for higher compression recovery as of monofilament has higher recovery \% than multifilament [8].

5. Anti-creasing finishing and anti-static finishing will be done after dyeing.

6. Anti-microbial finishes will be applied after dyeing so as to control the contamination.

\section{Expected Outcome}

1. The 3D structure itself will provide a smoother surface as well as create an acceptable micro-climate for the skin to breathe and perspire effectively to ensure a low friction coefficient. This includes high wicking and evaporation abilities as well as a reasonable air 
Citation: Shuvo II, Chakma K, Toutant D (2018) Prospect of 3D Warp Knitted Spacer Fabric and its Effect on Pressure Relieve for Reducing the Prevalence of Pressure Ulcers for Immobile Patients. J Textile Sci Eng 8: 335. doi: 10.4172/2165-8064.1000335

Page 4 of 4

permeability to ensure a proper temperature between the skin and fabric.

2. Having a higher density will greatly improve the thermal conductivity to keep the patient comfortable and the sheets drier from excess perspiration.

3. The compressibility of this fabric allows for a more even pressure distribution over the person's body. The compression recovery is high which increases the dimensional stability.

4. Anti-creasing finishes will help against wrinkling of the sheet which reduces the chance of creating additional high friction points.

5. Anti-microbial finishes will help control against contaminants.

\section{Benefits to North America}

The bed sheet can be outsourced to provide a cheaper product which is economical for North America. No extra machinery cost will be required because knitting machines can produce many different structures out of various materials with little to no modifications [12]. By creating a new fabric opens opportunities of starting a business and creating new jobs which would be taxed to benefit the North American economy. If the hospital does not want to completely implement these bed sheets at the very least, they should be made available for those who are immobile. These people are in the exact same position for hours at a time before being rotated by nurses. The bed sheet will allow for an easier time rotating the patient but will also protect against the development of pressure ulcers.

\section{References}

1. Levine JM (2016) Pressure Ulcers: An Underappreciated Public Health Issue.

2. Zhong W, Ahmad A, Xing MMQ, Yamada P, Hamel C (2008) Impact of textiles on formation and prevention of skin lesions and bedsores. Cutan Ocul Toxicol 27: 21-28.

3. Gerhardt LC, Strassle V, Lenz A, Spencer ND, Derler S (2008) Influence of epidermal hydration on the friction of human skin against textiles. Journal of Royal Society Interface 5: 1317-1328.

4. O'Neil CK (2004) Prevention and treatment of pressure ulcers. Journal of Pharmacy Practises 17: 137-148.

5. Basal G, Ilgaz S (2009) A functional fabric for pressure ulcer prevention. Textile Research Journal 79: 1415-1426.

6. Schario M, Lichterfeld A, Herfert H, Dobos G, Lahmann N et al. (2016) Effects of two different fabrics on skin barrier function under real pressure conditions. $\mathrm{J}$ Tissue Viability 26: 150-155.

7. Du Z, Wu Y, He L (2016) Determination of pressure indices to characterize the pressure-relief property of spacer fabric based on a pressure pad system. Textile Research Journal 86: 1443-1451.

8. Yip J, SP Ng (2008) Study of three-dimensional spacer fabrics: Physical and mechanical properties. Journal of Materials Processing Technology 206: 359364

9. Pan N, Sun G (2011) Functional textiles for improved performance, protection and health. Cambridge, UK: Wood head Publishing Limited and The Textile Institute.

10. Agrawal K, Chauhan N (2012) Pressure ulcers: Back to basics. Indian Journal of Plastic Surgery 45: 244-254.

11. Schilthizen S, Goijarts G (2015) Stretchable textile stay and transfer sheet. EP2921579 A1.

12. Leong KH, Ramakrishna S, Huang Z, Bibo GA (2000) The potential of knitting for engineering composites-a review. Elsevier Part 31: 197-220. 